Itinéraires Itinéraires

Littérature, textes, cultures

\title{
Florence 1926 : Solaria et la question de la modernité
}

Anne-Rachel Hermetet

\section{OpenEdition}

Journals

Édition électronique

URL : http://journals.openedition.org/itineraires/508

DOI : 10.4000/itineraires.508

ISSN : 2427-920X

Éditeur

Pléiade

Édition imprimée

Date de publication : 1 novembre 2009

Pagination : 123-134

ISBN : 978-2-296-10115-9

ISSN : 2100-1340

Référence électronique

Anne-Rachel Hermetet, « Florence 1926 : Solaria et la question de la modernité », Itinéraires [En ligne], 2009-3 | 2009, mis en ligne le 25 juin 2014, consulté le 19 avril 2019. URL : http:// journals.openedition.org/itineraires/508 ; DOI : 10.4000/itineraires.508

\section{(ब) $\Theta \Theta$}

Itinéraires est mis à disposition selon les termes de la licence Creative Commons Attribution - Pas d'Utilisation Commerciale - Pas de Modification 4.0 International. 


\title{
Florence 1926 : Solaria et la question de la modernité
}

\begin{abstract}
In the mid-1920s, Solaria, the Florentine journal, defined a literary program open towards Europe. By examining the modalities of the readings of foreign literatures it offered, we can analyze the ideological and aesthetic stakes of Solaria's project: what is at stake is at the same time to fight against the provincialism of Italian intellectual life and to offer a renewal of the genre of the novel in Italy. The analysis of the references made to Dostoievski, Gide, Joyce or Proust thus sheds light on the ambitions of the journal's contributors but also on the limitations of their enterprise.
\end{abstract}

Keywords : Solaria (periodical), literary journals, intellectual life, literary criticism, literary modernity - Italy (1919-1939)

Mots clés : Solaria (périodique), revues littéraires, vie intellectuelle, critique littéraire, modernité littéraire - Italie (1919-1939)

Il faudrait bannir le mot "modernité » de notre langage pour cause d'incitation au désordre intellectuel. Ou ne le convoquer qu'avec une fiche d'identité détaillée mentionnant le plus de signes particuliers possibles ${ }^{1}$.

Cette exhortation, salutaire, de Michel Décaudin invite à la prudence au moment de proposer une lecture de la revue florentine Solaria, présentée par la critique italienne contemporaine comme le paradigme de la revue moderniste dans l'entre-deux-guerres ${ }^{2}$. Ce sont ces « signes particuliers »

1. Michel Décaudin, «Modernité de Barnabooth», dans Jean Bessière (dir.), Valery Larbaud : la prose du monde, Paris, PUF, 1981, p. 93.

2. Voir, par exemple, Alberto Folin, Solaria, Letteratura, Campo di Marte, Treviso, Canova, 1973; Giuseppe Langella, Da Firenze all'Europa. Studi sul Novecento letterario, Milano, Vita e Pensiero, 1989, p. 145-220 ou, plus récemment, Roberto Ludovico, «Tra Europa e romanzo: Solaria e il fantasma James Joyce », dans Carla Gubert (dir.) Frammenti 
qu'on s'efforcera de dégager ici, en adoptant l'hypothèse selon laquelle, dans le contexte italien, l'accueil réservé aux littératures étrangères est un indice pertinent de modernité et que son examen permet de préciser les formes et les enjeux prêtés à celle-ci. De fait, le sentiment qui prévaut, parmi les intellectuels italiens des années 1920, est celui du provincialisme de la culture italienne. L'ambition, quelle que soit ensuite l'extension que l'on donne à ce mouvement, est d'élargir les horizons, de se déprendre de ce qui apparaît comme une étroite prison. Ce parti pris relève de questions tout à la fois esthétiques et idéologiques. Il s'agit de chercher des solutions à ce qui est perçu comme une crise de la littérature italienne et, en particulier, du roman, mais aussi de se situer par rapport au régime mussolinien ${ }^{3} . \mathrm{Ce}$ sont alors la lecture, le commentaire d'auteurs étrangers qui apparaissent comme un antidote sûr au risque d'enfermement et au discours de propagande. Solaria ne fait pas exception, qui propose à ses lecteurs un parcours dans les lettres européennes dont les modalités restent à préciser.

Il peut sembler paradoxal que Florence, cité perçue comme le berceau de la culture italienne, soit associée au milieu des années 1920 à la notion de modernité. Et pourtant, c'est entre collines et Arno qu'est née, en 1926, une revue qui devait incarner, aux yeux de ses contemporains comme de la critique ultérieure, les aspirations italiennes à la modernité. Son nom, Solaria, est, selon l'un de ses fondateurs, Bonaventura Tecchi, celui " d'une cité idéale, Soleil et Air, probablement, et aussi un je ne sais quoi de solitaire ${ }^{4} »$; il indique ainsi un des objectifs d'Alberto Carocci, directeur de la revue, et de ses collaborateurs : établir un lieu, fût-il idéal, en retrait de la vie réelle, une manière de tour d'ivoire où pourraient se déployer un discours critique et une pratique littéraire dégagés des contraintes idéologiques.

Cette posture s'explique dans le contexte italien : 1926 est une année charnière dans la vie culturelle de l'entre-deux-guerres en Italie. C'est, en effet, à cette date que Mussolini et son gouvernement entreprennent un processus de fascisation systématique des institutions. Si le régime avait pu se voir ébranlé après l'assassinat du député socialiste Matteotti, en 1924, et le retrait « sur l'Aventin » des députés de l'opposition, Mussolini a repris le contrôle du pays. En décembre 1925 et janvier 1926, une

d'Europa, Pesaro, Metauro, 2003, p. 39-59.

3. À cet égard, il convient de nuancer l'assimilation de l'européanisme à l'antifascisme : si les revues d'opposition au régime présentent toutes une composante européaniste, un projet comme celui de la revue 900, initié par Massimo Bontempelli ou les interventions critiques de Giovan Battista Angioletti s'inscrivent dans une perspective européaniste qui ne dénonce pas le fascisme.

4. " "Solaria" significa il nome di una città ideale, Sole e Aria, probabilmente, e insieme un che di solitario. » Cité par Sandro Briosi, Il problema della letteratura in Solaria, Milano, Mursia, 1976, p. 7 [sauf indication contraire, nous traduisons]. 
série de lois met en place la transformation de la démocratie en régime totalitaire $^{5}$ : Mussolini devient le seul titulaire du pouvoir exécutif et le gouvernement peut décider des lois sans en référer au Parlement. Le régime entreprend dans le même temps de réprimer efficacement l'opposition en contrôlant étroitement les associations et la presse et en exigeant la fidélité des fonctionnaires. C'est en 1926 également qu'Alfredo Rocco, alors ministre de la Justice, fait voter les «lois de défense de l'État», dites « lois fascistissimes », qui permettent de supprimer tout journal ou association soupçonné d'antifascisme et de détenir tout «subversif». Ce contrôle politique renforcé est relayé et soutenu par une propagande accrue et par la tentative de définir une culture fasciste. Les témoignages des intellectuels de l'époque attestent la sensation d'une atmosphère raréfiée, d'un rétrécissement des horizons, autour des thèmes développés par le discours officiel : grandeur de la patrie, exaltation de la romanité, de la famille, dénonciation des démocraties perçues comme décadentes. Dans un tel contexte, se donner un programme exclusivement littéraire, comme le fait Solaria, revient à se mettre en marge tout en préservant un espace de réflexion. Et lire des œuvres étrangères participe ipso facto de ce mouvement volontaire d'isolement, même si les mesures véritablement autarciques n'entrent en vigueur qu'au moment de la guerre d'Éthiopie et des sanctions votées contre l'Italie par la Société des Nations.

Revenant en 1958, à l'occasion de la publication d'une anthologie de Solaria, sur l'histoire de la revue, Alberto Carocci précise ainsi quelle fut sa place dans l'Italie du ventennio :

Je ne veux pas dire que Solaria, revue de littérature, eut une activité d'opposition politique au régime, ni une activité d'opposition sur le plan idéologique. [...] Beaucoup plus modestement et en acceptant l'isolement dans lequel la littérature officielle la reléguait (les lecteurs de Solaria ne furent jamais qu'une poignée; son tirage n'atteignait pas sept cents exemplaires; et je n'ai pas le souvenir qu'elle ait jamais eu l'honneur d'être citée par les journaux du temps), elle joua tout au plus un rôle d'objecteur de conscience. La littérature officielle célébrait le génie italien, la suprématie de l'Italie, les gloires de la lignée et toutes les pages de Solaria exprimaient la conviction que la littérature italienne contemporaine n'était qu'une province de la plus vaste littérature européenne, et pas même sa province la plus magnifique. La littérature officielle affirmait qu'existaient, au sein de l'Italie, toutes les prémisses et tous les résultats auxquels une littérature moderne pouvait prétendre; et Solaria reconnaissait humblement que les expressions les plus originales de la littérature moderne s'étaient épanouies ailleurs, qu'elles s'appellent Proust, Joyce ou Kafka, et affirmait que les écrivains italiens étaient assurément des voix dignes de considération mais

5. Voir Pierre Milza et Serge Berstein, Le Fascisme italien 1919-1945, Paris, Seuil, 1980, p. 141-143. 
qu'ils constituaient une partie, et une partie seulement, d'un plus vaste concert européen ${ }^{6}$.

Cette évocation d'Alberto Carocci souligne la dimension européenne de Solaria mais aussi la précarité de ses conditions matérielles d'existence : la revue ne dispose pour toute source de financement que du montant des abonnements et de contributions volontaires de ses collaborateurs. L'analyse de Carocci met aussi en évidence les limites du projet solarien: il n'est pas toujours possible, en effet, de garder une position d' « objecteur de conscience ». Dans les années 1930, les équilibres se modifient dans la mesure où le régime fasciste jouit d'un large consensus intérieur - dont une des étapes majeures a été, en février 1929, la signature des accords du Latran qui mettent fin, au moins pour un temps, aux tensions entre le pouvoir politique et l'Église catholique - et d'une solide reconnaissance à l'étranger. Le groupe de Solaria se divise alors autour de la question de l'engagement: une partie des collaborateurs, autour d'Alessandro Bonsanti, co-directeur de la revue à partir de 1930, entend maintenir le débat sur le seul terrain littéraire et donne naissance en 1937 à une autre revue, Letteratura; d'autres, avec Alberto Carocci, souhaitent un engagement dans la cité non plus idéale mais temporelle. Dans le même temps, la revue rencontre de grosses difficultés matérielles, le numéro daté de mars-avril 1934 a été saisi pour pornographie ${ }^{7}$. Solaria survit de fin 1934 au printemps 1936 (la dernière livraison porte la mention « an IX, $\mathrm{n}^{\circ}$ 5-6, septembre-décembre 1934-XII, publié le 31 mars $1936 »$ ) avant de mettre un terme à une activité, jugée dès 1934 « inutile» par Alberto Carocci ${ }^{8}$.

Le programme littéraire de Solaria se dessine dès l'éditorial inaugural de la revue :

6. Alberto Carocci, introduction à Enzo Siciliano (dir.), Antologia di « Solaria », Milano, Lerici, 1958, p. 10 : « Non intendo dire che "Solaria", rivista di letteratura, svolse un'attività di opposizione politica al regime, e neppure un'attività di opposizione sul piano ideologico. [...] Essa, molto più modestamente, e con l'accettare l'isolamento in cui la letteratura ufficiale la relegava (i lettori di "Solaria" furono sempre quattro gatti; la sua tiratura non raggiungeva le 700 copie; né ricordo che essa ricevesse mai l'onore di esser citata dai giornali del tempo) svolse tutt'al più una funzione di obiettore di coscienza. La letteratura ufficiale celebrava il genio italico, il primato d'Italia, le glorie della stirpe; e tutte le pagine di "Solaria" manifestavano la persuasione che la letteratura italiana contemporanea non era che una provincia della più vasta letteratura europea, e neanche la provincia più splendida. La letteratura ufficiale affermava che in seno all'Italia esistevano tutte le premesse e tutti i risultati ai quali una cultura moderna poteve ambire; e "Solaria" riconosceva umilmente che le espressioni più originali della letteratura moderna erano fiorita altrove, si chiamassero esse Proust o Joyce o Kafka, e affermava sì che anche gli scrittori italiani erano voci degne di considerazione, ma che erano parte e soltanto parte di un più vasto colloquio europeo. » 7. En raison de la publication d'une nouvelle d'Enrico Terracini, « Le figlie del generale », et du roman d'Elio Vittorini, Il garofano rosso, qui paraissait en feuilleton dans la revue. 8. Lettre à Carlo Ginzburg du 5 mars 1934, dans Giuliano Manacorda (dir.), Lettere a Solaria, Roma, Editori Riuniti, 1979, p. 486-487. 
« Solaria » naît sans programme précis, mais nantie d'une hérédité qui n'est pas méprisable. [...]

Ceux qui ont l'habitude de feuilleter les revues littéraires italiennes encore lisibles reconnaîtront parmi nous plus d'un visage connu, justement parce que nous voulons vivre dans un air de libertés et d'habitudes déjà éprouvées. Mais nous voudrions rapidement, dans cette lumière qui semblera tout de suite familière à beaucoup, nous faire reconnaître comme un groupe.

Nous n'idolâtrons ni les effets de style ni les purismes exagérés et si l'un d'entre nous sacrifie le rythme harmonieux d'une phrase et peut-être la correction de la langue parce qu'il tente de donner vie à un art particulièrement dramatique et humain, nous lui pardonnons à l'avance avec passion. Pour nous, en somme, Dostoïevski est un grand écrivain. Mais nous ne pardonnerons pas, même à nos hôtes fraternels, les licences qui ne seraient pas pleinement justifiées et en cela nous nous sentons enfants de La Ronda ${ }^{9}$.

Les deux noms cités dans les dernières lignes indiquent la complexité et les enjeux du projet. Le groupe de Solaria se réclame, en effet, explicitement, à la fois de $L a$ Ronda et de Dostoïevski ${ }^{10}$ et il n'est pas évident que les deux références puissent être conciliées. La Ronda, revue romaine parue entre 1919 et 1923, s'était donné un programme de retour à l'ordre, loin des excès prêtés aux avant-gardes, et avait proposé une réévaluation de la tradition nationale, sous le signe de Leopardi, génie tutélaire des collaborateurs de la revue. Les pages de la revue mêlent de brèves proses, souvent poétiques, entre récit et essai ${ }^{11}$, qualifiées en italien de "prosa $d^{\prime}$ arte $^{12} \gg$ et des commentaires critiques portant sur la littérature italienne et sur les littératures étrangères, française en particulier. L'ensemble est marqué par un grand souci de rigueur et un intérêt constant pour les questions stylistiques. Les collaborateurs de La Ronda affirment la nécessité

9. Note rédactionnelle anonyme, Solaria, I, n 1, janv. 1926, p. 3-4: «"Solaria" nasce senza un programma preciso e con qualche non spregevole eredità. [...] / Chi ha l'abitudine di sfogliare le riviste letterarie italiane ancora leggibili scorgerà tra noi più di un viso non ignoto, giustappunto perché vogliamo vivere in un'aria di libertà e di consuetudini già provate. Ma presto, in questa luce che a molti parrà subito famigliare, voremmo farci riconoscere come un gruppo. / Non siamo idolatri di stilismi e purismi esagerati e se tra noi qualcuno sacrifica il bel ritmo di una frase e magari la proprietà del linguaggio nel tentativo di dar fiato a un' arte singolarmente drammatica e umana gli perdoniamo in anticipo con passione. Per noi, insomma, Dostojevskij è un grande scrittore. Ma non perdoneremo nemmeno ai fraterni ospiti le licenze che non sieno pienamente giustificate e in questo ci sentiamo rondeschi. »

10. Solaria s'inscrit également dans la descendance du Baretti, revue turinoise fondée par Piero Gobetti en 1924, dans une perspective explicite de résistance culturelle au régime fasciste.

11. Un bon exemple en est donné par le recueil Pesci rossi d'Emilio Cecchi, même si les textes qui le composent sont, en grande majorité, antérieurs aux années de La Ronda.

12. Voir à ce sujet Carla Gubert, Un mondo di cartone. Nascita e poetica della prosa d'arte nel Novecento, Pesaro, Metauro, 2003. 
d'une autonomie de la culture, hors de tout engagement public et cherchent à définir une morale littéraire et artistique ${ }^{13}$.

Deuxième nom cité, celui de Dostoïevski. Il n'est pas, loin s'en faut, un auteur apprécié du groupe de La Ronda qui fait de lui le symptôme d'une époque malade. Ardengo Soffici et Riccardo Bacchelli voient en lui une incarnation de l' " âme russe » dans ce qu'elle a de primitif, de profondément étranger pour un Occidental : ainsi Soffici peut-il affirmer que ses personnages constituent une galerie des « monstruosité[s] du désordre mental », de «toutes les maladies et toutes les tares dont souffre la malheureuse humanité et, en particulier, le peuple russe ${ }^{14} »$, de l'épilepsie à l'alcoolisme ou à la folie. Dans la lignée de Melchior de Vogüé, qui qualifiait le roman dostoïevskien de « confus, mal bâti, ridicule », Soffici dénie toute qualité littéraire à un romancier qu'il a lu dans la traduction française ou italienne :

Dostoïevski, qui ressentait et concevait avec une force extrême, écrivait mal. Il écrivait dans une langue pauvre et négligée. Il n'avait ni le sens, ni le goût, ni le respect du mot qui dépeint, du mot harmonieux, qui, seul, peut animer pour l'éternité les visions de la fantaisie. Il écrivait comme un auteur de roman-feuilleton ${ }^{15}$.

Le jugement de Soffici n'est pas seulement littéraire : le critique, qui fut avant la Première Guerre mondiale un auteur d'avant-garde, ami d'Apollinaire, de Picasso et de Max Jacob, voit une incompatibilité profonde entre la littérature russe, dans son ensemble, et le monde d'après-guerre, dans la mesure où elle est à ses yeux "l'incarnation la plus notable et la plus certaine $»$ des erreurs modernes ${ }^{16}$, soulignant ainsi une évolution intellectuelle personnelle. Riccardo Bacchelli, quant à lui, tranche en faveur de Tolstoï dans le parallèle - classique - qu'il dresse entre les deux romanciers parce qu'à ses yeux, le « génie » de Dostoïevski est «tendancieux », " difforme et disproportionné », « sardonique et inquiet ${ }^{17} »$, « [sa] vocation d'artiste [...] ne parvint jamais à vaincre en lui cette autre vocation de prédicateur et de visionnaire intellectuel qui gâchait

13. Voir notre « Une arrière-garde à l'italienne? La Ronda », dans William Marx (dir.), Les Arrière-gardes au XXe siècle, Paris, PUF, 2004, p. 203-213.

14. Ardengo Soffici, «Osservazioni sulla letteratura russa », La Ronda, IV, $\mathrm{n}^{\circ} 3-4$, marsavril 1922, p. 205 : «Tutte le malattie e tutte le tare onde soffre la sciagurata umanità e specialmente il popolo russo. »

15. Ibid. : «Dostoewskij, il quale sentiva e concepiva con forza estrema, scriveva male. Scriveva con lingua povera e sciatta. Non aveva né il senso, né il gusto, né il rispetto della parola pittrice ed armonica, cui solo è concesso animare per l'eternità le visioni della fantasia : scriveva come un romanziere d'appendice. »

16. Ibid. : « la più notevole e certa incarnazione. »

17. Ricardo Bacchelli, « Paradosso su Tolstoj e Dostojewsky », La Ronda, IV, ${ }^{\circ}$ 12, décembre 1923, p. 818 : « Tendenzioso genio disgraziato e madornale, sardonico ed inquieto. » 
ses meilleures conceptions ${ }^{18} \gg$. Les rédacteurs de La Ronda proposent ainsi à leurs lecteurs l'image d'un Dostoïevski inquiétant, cas clinique plus que romancier. Les collaborateurs de Solaria renversent cette analyse en faisant de l'inquiétude que suscitent les personnages dostoïevskiens le signe même de l'homme moderne, en se situant dans la lignée de Jacques Rivière et d'André Gide ${ }^{19}$. Ils cherchent, dans leur lecture de Dostö̈evski, des éléments de réponse à des questions non seulement formelles mais aussi morales et s'attachent chez lui à l'expression d'une complexité humaine profondément ressentie.

Se réclamer tout à la fois de La Ronda et de Dostoïevski est donc révélateur d'une tension féconde qui donne lieu à l'une des expériences littéraires et revuistes les plus intéressantes de la première moitié du $\mathrm{xx}^{\mathrm{e}}$ siècle. D'un côté, le discours de Solaria s'épanouit sous le signe de la rigueur formelle et, si expérimentation il y a, elle se situe davantage dans le champ de la connaissance de l'individu que dans celui de la langue. Solaria, en effet, a hérité de La Ronda une défiance profonde à l'égard des avant-gardes, jugées coupables d'avoir permis, par la destruction des structures qu'elles prônaient, le chaos de la Première Guerre mondiale. De l'autre côté, s'amorce dans la revue une recherche de grande ampleur sur le genre romanesque. Dans l'historiographie littéraire italienne, Solaria est ainsi reconnue comme le lieu du passage du fragment, forme privilégiée dans La Ronda, au roman. Ses collaborateurs multiplient les recherches théoriques et pratiques dans le domaine de la prose narrative : c'est dans Solaria qu'est rendu hommage à Italo Svevo en $1928^{20}$ et à Federigo Tozzi en $1930^{21}$, dans Solaria qu'on peut lire des pages de Carlo Emilio Gadda ou d'Elio Vittorini, pour ne citer qu'eux, dans Solaria toujours que sont commentées les parutions italiennes ou étrangères les plus récentes.

En effet, le retour au roman, après les temps de la "prosa d'arte», exige de porter le regard vers l'étranger, dans la mesure où la production italienne semble s'être interrompue après le réalisme et les œuvres de D'Annunzio. Il s'agit désormais de définir des formes susceptibles de rendre compte d'un monde qui change et d'une perception différente de l'individu. L'attention des collaborateurs de Solaria se porte surtout sur le roman d'analyse car la modernité telle qu'ils la définissent s'identifie à l'exploration minutieuse de la psyché, dessinant la carte d'une Europe littéraire tout entière vouée à l'étude du cœur humain. C'est ainsi qu'ils réunissent Proust, Gide, Joyce, Virginia Woolf et, en Italie, Italo Svevo dans

18. Ibid. : « la vocazione d'artista di Dostojewsky non riuscì mai a vincere in lui quell'altra del predicatore e del visionario intellettuale, che gli sciupava le sue migliori concezioni. "

19. Sur la réception de Dostoïevski, voir Sergia Adamo, "Il Dostoevskij di "Solaria" ", Comparatistica, 1996, p. 61-78.

20. Solaria, IV, n 3-4, mars-avril 1928.

21. Solaria, V, nº 5-6, mai-juin 1930. 
un même concert, comme autant de voix ayant mené cette exploration ${ }^{22}$. Cette perspective résolument européenne ressort clairement du numéro d'hommage à Svevo, qui réunit des contributions en quatre langues : on peut y lire, entre autres, des réflexions de Benjamin Crémieux, Valery Larbaud, Paul-Henry Michel, traducteur du romancier, Adrienne Monnier, dont on connaît le rôle dans la publication d'Ulysses, Philippe Soupault, de James Joyce et d'Ilia Ehrenbourg ainsi que de la jeune critique militante italienne, puisque Giacomo Debenedetti, Leo Ferrero, Eugenio Montale ou Sergio Solmi, pour ne citer que quelques noms, participent à l'hommage. On mesure ainsi l'importance accordée au romancier triestin, qui n'est pas resitué dans l'histoire littéraire italienne mais bien inséré dans l'évolution du roman en Europe. C'est par cette voix venue d'une marge du royaume que le roman italien doit entrer dans la modernité et le fait que l'œuvre de Svevo ait été reconnue à l'étranger, par Joyce, par Larbaud, par Crémieux, avant de l'être en Italie ne vient que confirmer, aux yeux de ses promoteurs, la pertinence de l'analyse solarienne.

La Nouvelle Revue française joue un rôle considérable de passeur dans ce processus ${ }^{23}$. Outre un lien privilégié avec Crémieux, qui commente la littérature italienne contemporaine dans la revue parisienne, celle-ci propose aux critiques florentins un accès à la littérature en train de se faire mais aussi le modèle d'une micro-société vouée à la littérature, démontrant ainsi que le projet solarien est viable. Il n'est pas surprenant, alors, que $L a$ $N R F$ fournisse aux solariens une part substantielle des œuvres recensées, qu'il s'agisse d'auteurs publiant dans ses pages ou d'œuvres faisant l'objet de recensions: en 1926, par exemple paraissent un éloge de Jacques Rivière en mars ${ }^{24}$, un article sur l'œuvre de Gide, centré sur Les Cahiers d'André Walter en mai ${ }^{25}$, une longue analyse des proses de Paul Valéry en juillet et septembre ${ }^{26}$ et, au fil des années, des interventions consacrées

22. Voir à ce sujet Giuseppe Langella, Da Firenze a l'Europa. Studi sul Novecento letterario, Milano, Vita e Pensiero, 1989, p. 166-175.

23. Voir à ce sujet les travaux de Gilbert Bosetti dont : «Les modèles "NRF" des récits de "Solaria" ", Novecento, Cahiers du CERCIC, n 5, 1985, p.83-157; « Les lettres françaises sous le fascisme. Le culte de la "N.R.F." dans l'entre-deux-guerres face à la francophobie fasciste ", Mélanges de l'École française de Rome, 1986, vol. 98, n 1, p. 383-432; «L'Axe Paris-Florence dans la culture italienne de l'entre-deux-guerres » dans Paris et le phénomène des capitales littéraires : carrefour ou dialogue des cultures, actes du premier congrès international du C.R.L.C., vol. II, Paris, Presses de l'Université de Paris-Sorbonne, 1986, p. 789-802; «Le culte de la N.R.F. et le renouveau du roman italien dans l'entredeux-guerres », dans Novecento, Cahiers du CERCIC, n 7, Grenoble, 1986, p. 15-72; « Le rayonnement des écrivains de la "NRF" en Italie (1920-1950) », Novecento, Cahiers $d u$ CERCIC, $\mathrm{n}^{\circ} 11,1989$, p. 65-82.

24. Giovanni Titta Rosa, «Elogio di Rivière », Solaria, I, n 3, mars 1926, p. 35-38.

25. Rafaello Franchi, «I Quaderni di Gide », Solaria, I, n 5, mai 1926, p. 31-36.

26. Pierro Burresi, «Commento alle prose di Paul Valéry », Solaria, I, n 7-8, juillet-août 1926, et I, nº 9-10, sept.-oct. 1926. 
à Georges Duhamel ${ }^{27}$, Mauriac ${ }^{28}$, Paulhan ${ }^{29}$ ou Chardonne ${ }^{30}$ pour ne citer que quelques noms. La modernité selon Solaria passe ainsi par la France et par le filtre des choix opérés par Jean Paulhan et, avant lui, par Jacques Rivière. L'influence de ce dernier en Italie au début des années 1920 est remarquable et elle contribue à expliquer la prédominance du roman d'analyse comme modèle. À la recherche du temps perdu est ainsi lu comme une longue introspection dévoilant peu à peu les secrets enfouis dans le subconscient du narrateur. Et il n'est pas anodin qu'Alberto Carocci ait eu le projet, inabouti, de traduire au début des années 1930, La Princesse de Clèves pour l'éditeur Treves ${ }^{31}$.

Plus largement, les comptes rendus sont, en très grande majorité (près des deux tiers), consacrés à des œuvres françaises, suivies de très loin par les littératures de langue allemande, la littérature russe et les littératures anglaise et irlandaise. La prédominance de la littérature française s'explique assurément par des raisons linguistiques puisque la pratique du français était (encore) courante dans les milieux cultivés; elle est accentuée, dans le groupe de Solaria, par la fascination qu'exercent une ville, Paris, et, donc, La Nouvelle Revue française. Toutefois, un parcours dans les comptes rendus tout comme dans les textes étrangers publiés dans Solaria invite à s'interroger sur la place effective des œuvres identifiées comme « modernes » dans la revue. Il semble qu'il y ait un décalage entre les textes donnés à lire dans Solaria, les recensions critiques et les mentions faites d'auteurs étrangers. La revue propose peu, en effet, de textes étrangers, en langue originale ou en traduction: "Arkas e Arete» du finlandais Emil Liliacus $^{32}$, un texte de Synge, «La cavalcata al mare » (Riders to the Sea), traduit par Joyce et Nicolò Vidacovich ${ }^{33}$, « Canto di Simeone », traduction de «A Song for Simeon», de T. S. Eliot par Eugenio Montale ${ }^{34}$, des poèmes d'Ossip Mandelstam, traduits par Renato Poggioli ${ }^{35}$, deux poèmes de Rilke, traduits par Giansiro Ferrata ${ }^{36}$. Si les choix sont remarquables, le bilan reste quantitativement modeste et ne permet pas aux lecteurs un accès régulier aux œuvres étrangères.

27. Giansiro Ferrata, c. r. de Georges Duhamel, Le Voyage de Moscou, Solaria, III, n 1 , janv. 1928.

28. Giansiro Ferrata, c. r. de François Mauriac, Destins, Solaria, III, n 4, avril 1928.

29. Alado Capasso, c. r. de Jean Paulhan, Le Guerrier appliqué, Solaria, V, n 12, déc. 1930.

30. Aldo Capasso, c. r. de Jacques Chardonne, Eva ou le journal interrompu, Solaria, VI, $\mathrm{n}^{\circ} 3$, mars 1931.

31. Voir Giuseppe Langella, op. cit., p. 171.

32. Solaria, III, n 6 , juin 1928.

33. Solaria, IV, $\mathrm{n}^{\circ}$ 9-10, sept.-oct.1929.

34. Solaria, IV, n 12, déc. 1929.

35. Solaria, VI, ${ }^{\circ} 1$, janv. 1931.

36. Solaria, VIII, nº 8-10, août-oct. 1933. 
Les comptes rendus sont, en nombre, plus importants mais il serait faux de croire que les auteurs cités comme références ont tous fait l'objet d'études développées. Ainsi, Proust est, paradoxalement, un grand absent de Solaria: la publication du Temps retrouvé, en 1927, n'y donne lieu à aucune recension, non plus d'ailleurs que dans aucune autre revue littéraire $^{37}$. Et Proust n'est présent que par le compte rendu des essais critiques, certes publiés aux éditions de Solaria, que lui consacre Giacomo Debenedetti, qui joua un rôle considérable dans la diffusion de son œuvre en Italie $^{38}$. On peut ainsi mesurer le décalage qui peut se produire entre les lectures privées d'un auteur et les publications critiques portant sur son œuvre, puisqu'on sait, par d'autres sources, que La Recherche a été abondamment lue et méditée. Anna Dolfi l'a rappelé à juste titre, en dressant la longue liste des romanciers ayant reconnu une influence proustienne sur leur œuvre, liste où figurent, entre autres, des collaborateurs de Solaria comme Elio Vittorini ou Cesare Pavese ${ }^{39}$.

De la même façon, l'accueil réservé à l'œuvre de Joyce est plus que modeste : il intervient en son nom propre dans le numéro d'hommage à Italo Svevo, son $\mathrm{ami}^{40}$; la revue publie, en 1929, on l'a vu, la traduction italienne d'un acte de John Millington Synge, Riders to the Sea, qu'il a effectuée avec son ancien élève Nicolò Vidacovich ${ }^{41}$ mais aucun de ses collaborateurs ne consacre une recension à son œuvre. Lorsqu'une critique est publiée, il s'agit, en mars 1930, d'un chapitre de l'essai de Stuart Gilbert, James Joyce's Ulysses. On peut s'interroger devant une telle prudence, dans une revue où les interventions critiques, souvent précises, ne manquent pas. On peut sans doute l'attribuer à des questions linguistiques (et il est vraisemblable que la traduction française a été plus lue que le texte original, d'autant qu'elle était accompagnée de la médiation de Valery Larbaud) mais elles ne suffisent pas à expliquer ce retrait. On notera aussi la séparation entre traductions de l'œuvre et discours critique;

37. On peut juste mentionner une note de Giacomo Prampolini, dans la revue de presse qu'il assure pour l'hebdomadaire La Fiera Letteraria (Giacomo Prampolini, « La stampa estera : l'addio di Proust» (avec des extraits traduits en italien), La Fiera Letteraria III, $\mathrm{n}^{\circ} 40,2$ octobre 1927, p. 7).

38. Aldo Capasso, « Debenedetti e Proust », Solaria, VI, n 1, janv. 1930. Sur Debenedetti et Proust, voir Viviana Agostini-Ouafi, « La critique de Proust chez Giacomo Debenedetti (1925-1946) » dans Viviana Agostini-Ouafi (dir.), Proust en Italie, Transalpina, $\mathrm{n}^{\circ}$ 7, 2004, p. 41-60 et notre «Aux yeux de la critique italienne, À la recherche $d u$ temps perdu est-il un "roman français" (1919-1925)?», ibid., p. 15-25.

39. Voir à ce sujet Anna Dolfi, « Proust, il proustismo e l'incidenza proustiana nella cultura italiana del Novecento. Prodromi di una ricerca », Franco-italica, serie contemporanea, vol. $2, n^{\circ} 4,1993$, p. 21-40.

40. Solaria, IV, $\mathrm{n}^{\circ}$ 3-4, mars-avril 1928.

41. Solaria, IV, $n^{\circ}$ 9-10, sept.-oct. 1929, p. 3-6. Voir Ornella De Zordo, « James Joyce nelle riviste fiorentine tra le due guerre », Rivista di letterature moderne e comparate, vol. 36, $\mathrm{n}^{\circ} 3$, juillet-sept. 1983, p. 254. 
cette répartition n'est pas sans analogie avec la réception française de l'œuvre de Joyce puisqu'on retrouve dans Solaria le décalage entre un discours critique plutôt ouvert et une pratique littéraire sensiblement plus traditionnelle dans ses formes qu'on peut observer dans $L a N R F$. En effet, si l'étude de Valery Larbaud sur Joyce paraît en janvier 1922 dans La NRF, Jacques Rivière, alors directeur de la revue, refuse de publier des extraits de la traduction d'Ulysses, qui trouvent place dans la première livraison de Commerce en $1924^{42}$. Parallèlement, la première publication d'extraits d'Ulysses en Italie se fait dans la première livraison de 900 (Novecento, c'est-à-dire « Vingtième siècle »), qui publie, à l'automne 1926, un extrait de l'épisode de Calypso, dans la traduction française d'Auguste Morel et Valery Larbaud ${ }^{43}$.

Pareil traitement fait sens tout comme celui de l'œuvre de Proust : tout se passe comme si le nom de James Joyce, celui de Proust, mentionnés à plusieurs reprises, fonctionnaient comme des indicateurs de modernité. Citer Joyce ou Proust, en d'autres termes, revient à s'assurer une forme de légitimité intellectuelle en prenant place ipso facto dans une constellation où figurent aussi Gide ou Valéry. Ainsi, plus que les articles, ce sont les mentions, au sens de Karl Rosengren ${ }^{44}$ qui dressent une cartographie de la modernité et y situent le projet solarien. Il n'en demeure pas moins que la perspective européenne que se fixe Solaria est indéniable et elle a été perçue comme telle à l'époque de la parution de la revue. Parler de Gide, de Svevo, de Proust a attiré à ses collaborateurs des attaques qui mêlent l'accusation de philosémitisme à celle de « corydonisme ${ }^{45}$ ». À l'intérieur même de la rédaction, des critiques comme Giansiro Ferrata et Elio Vittorini plaident pour un élargissement aux littératures étrangères et provoquent une crise dont s'inquiète le directeur, Alberto Carocci. Ainsi peut-il écrire, en 1930, à Bonaventura Tecchi : « Nos très bons, très valeureux et très intelligents jeunes amis commencent à exagérer un peu. Ils joycisent, proustisent, valérysent, ils brouillent les cartes avec un zèle qui passe le nécessaire. ${ }^{46} »$

42. Voir à ce sujet Régis Salado, «Les voies de l'hospitalité : la première réception française de l'œuvre de James Joyce » dans Anne-Rachel Hermetet (dir.), Les romanciers français lecteurs et spectateurs de l'étranger (1920-1950), Lille, CeGes, coll. «UL3 », 2004, p. 50. 43. Sur la première réception de l'œuvre de Joyce en Italie, voir Roberto Ludovico, « Tra Europa e romanzo : Solaria e il fantasma James Joyce », dans Carla Gubert, op. cit., p. 3959.

44. Voir Karl Erik Rosengren, Sociological Aspects of the Literary System, Lund, 1968. Une mention est constituée par «le nom d'un auteur de fiction (narrative, dramatique et poétique), d'autobiographie, d'essais d'intérêt général, ou toute allusion à un tel écrivain » dans " un compte rendu d'un ouvrage de fiction récemment publié » et écrit par un autre auteur (op. cit., p. 26 [nous traduisons]).

45. Voir à ce sujet Giuseppe Langella, op. cit., p. 151-161.

46. Lettre de Carocci à Bonaventura Tecchi, $1^{\text {er }}$ avril 1930, citée par Giuliano Manacorda, 
Toutefois, on notera que le discours tenu sur les œuvres étrangères reste prudent, tout comme restent modérées les expérimentations formelles tentées par les jeunes auteurs publiés dans la revue. On peut penser que ce ton est, pour une part, issu de la défiance à l'égard des expérimentations langagières des avant-gardes que les solariens ont hérité de La Ronda, tout comme du refus de la parole officielle, emphatique et réductrice. Surtout, la modernité, pour les solariens, réside davantage dans l'approche de l'individu, la révélation sans fard de ses interrogations existentielles et de ses abîmes intimes. C'est en ce sens qu'ils convoquent Dostoïevski, Gide ou Proust, comme hérauts d'une exploration qui se dégage des normes de la morale courante et rend compte de l'inquiétude de l'homme contemporain.

\author{
Anne-Rachel Hermetet \\ Université d'Angers - CERIEC
}

\footnotetext{
« Carteggi solariani Carocci, Ferrata, Bonsanti a Tecchi », dans Gioacchino Paparelli et Sebastiano Martelli (dir.), Letteratura fra centro e periferia, Napoli/Roma, Edizioni Scientifiche Italiane, 1987, p. 1019 : « I nostri ed ottimi e valorosissimi e intelligentissimi giovani amici incominciano un pochino a esagerare. Joysseggiano, prousteggiano, valéryggiano, arrufan le carte in tavola con uno zelo superiore a ogni necessità. » Carocci choisit ensuite de confier la co-direction de la revue à Alessandro Bonsanti, moins « marqué » que Ferrata dans le débat européaniste.
} 